\title{
Mechanism of Heat Transfer Enhancement in the Core Flow of a Tube and Its Numerical Simulation
}

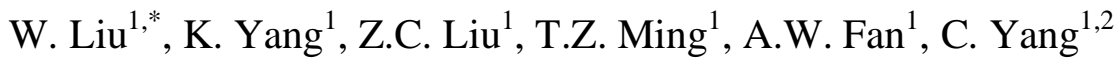 \\ ${ }^{1}$ School of Energy and Power Engineering, Huazhong University of Science and Technology, Wuhan 430074, China \\ ${ }^{2}$ Department of Mechanical Engineering, Shizuoka University, 3-5-1 Johoku, Hamamatsu, 432-8561, Japan
}

\begin{abstract}
The principle of heat transfer enhancement in the core flow of a tube has been proposed, in this paper, to make fluid temperature uniform in the core region of a tube and decrease flow resistance, which is different from heat transfer enhancement in the boundary flow of a tube. Two new models, representing heat transfer enhancement in the laminar and turbulent tube flow, have been established and numerically analyzed. Theoretical and numerical results indicate that heat transfer enhanced components designed according to the principle proposed in this paper will be benefit for increasing convective heat transfer coefficient, reducing flow resistance and raising the $P E C$ value of a heat transfer enhanced tube. The presented principle, therefore, may help developing new type of heat transfer unit and designing heat exchanger with high heat transfer coefficient and low flow resistance.
\end{abstract}

Keywords: Heat transfer enhancement, core flow, tube, flow resistance.

\section{INTRODUCTION}

Heat exchangers, which have been and are being widely used in many fields like electric power, chemical industry, metallurgy, steel, refrigeration, air-conditioning and so on, are indispensable devices to transfer energy. Therefore, it is essential to develop theory and technique about heat transfer enhancement in the tube flow to raise the performance of a heat exchanger. Generally speaking, tube flow can be divided into two parts, boundary flow and core flow. The boundary flow is a fluid region near the wall in the tube, beyond which in the tube the core flow is defined. In order to enhance heat transfer in the tube flow, commonly used methods $[1,2]$ include increasing fluid velocity, disturbing boundary layer, extending heat transfer surface, changing physical properties of heat transfer surface and so on. Correspondingly, heat transfer enhanced tubes include [3-6] spiral grooved tube, longitudinal troughed tube, corrugated tube, inner-finned tube, spiral-ribbed tube, micro-ribbed tube and so on. These applications are mainly considered to effectively design and improve heat transfer surface in the boundary flow. Moreover, those improved surfaces dominate convective heat transfer between the fluid and the tube wall. Therefore, this kind of method can be called surface-based heat transfer enhancement, or heat transfer enhancement in the boundary flow.

The recent work $[7,8]$ emphasizes that there exists a core region in the tube flow, in which fluid-based heat transfer enhancement can be considered, different from surface-based heat transfer enhancement in a tube. If some proper measures are adopted in this region, the effect of heat transfer en-

*Address correspondence to this author at the School of Energy and Power Engineering, Huazhong University of Science and Technology, Wuhan 430074, China; Tel: +86 27 87542618; E-mail: w_liu @ hust.edu.cn hancement can be obtained without increasing too much flow resistance. Though there are numerous researches and applications in the aspects of the tube inserts [9-18], the fluid near the tube wall is greatly influenced by the tube inserts which bring great fluid friction and momentum dissipation. More importantly, as flow resistance increases, those insert techniques would have negative impacts on the overall performance of heat transfer unit or heat exchanger.

\section{PRINCIPLE AND METHOD}

In the tube side of a heat exchanger, increasing fluid velocity will lead to higher shear stress, intensifying fluid disturbance in the boundary flow will result in more dissipation of fluid momentum, and enlarging continuously extended surface will cause more frictional resistance and viscosity dissipation. Thus, the flow resistance will be increased by adopting these techniques. If the flow resistance is overlarge, the fluid velocity will become small, which may weaken convective heat transfer between the fluid and the surface. Therefore, reducing flow resistance will be one of the key factors of developing heat transfer enhancement techniques with lower power dissipation.

\subsection{Principle of Heat Transfer Enhancement in the Core Flow of a Tube}

In order to achieve the effect of heat transfer enhancement, a method that can be taken into account is inserting heat transfer component in the core flow of a tube. The basic consideration of heat transfer enhancement in this case is to uniform fluid temperature in the core flow and create an equivalent thermal boundary layer with relatively larger temperature gradient near the wall, which helps to enhance convective heat transfer between the fluid and the tube wall. Meanwhile, the flow resistance will not increase remarkably. The forming of an equivalent thermal boundary layer near the tube wall could be independent of the velocity boundary 
layer. For the purpose of reducing flow resistance, the boundary layer with relatively larger velocity gradient should be avoided. Therefore, for improving the overall heat transfer performance or raise the $P E C$ value, the increase in heat transfer may surpass the increase in flow resistance after taking proper measure in the core flow region. To meet this requirement, Liu et al. [7, 8] proposed a principle of heat transfer enhancement in the core flow, which is mainly expressed as (1) strengthening temperature uniformity in the core flow; (2) increasing fluid disturbance in the core flow; (3) reducing surface area of heat transfer component in the core flow; (4) decreasing fluid disturbance in the boundary flow.

Based on the principle, when developing a technique of heat transfer enhancement in the core flow of a tube, we should avoid strongly disturbing the fluid near the wall. In addition, the contact between the heat transfer component and the tube wall should be prevented, and the function of this component without contacting heat source or heat sink is just to disturb the fluid or uniform the fluid temperature. As the component for heat transfer enhancement in the core flow does not conduct heat from the tube wall, no convective heat transfer occurs at any point between the component surface and the fluid. So we can define that (1) surface-based heat transfer enhancement is one type that convective heat transfer occurs between the wall surface and the fluid; (2) fluid-based heat transfer enhancement is another type that there is no convective heat transfer between the component surface and the fluid, which is so-called heat transfer enhancement in the core flow.

\subsection{Method of Heat Transfer Enhancement in the Core Flow}

According to the requirement of heat transfer enhancement in the core flow of a tube, The components of heat transfer enhancement can be designed as two types, uniform temperature type and disturbing flow type. The common objective of the two is to uniform fluid temperature in the core flow of a tube. The former enhances heat transfer rate by increasing effective heat conductivity of heat transfer medium, and the latter mixes the fluid in the core flow intensively to realize uniform fluid temperature. Additionally, the heat transfer components should be distributed discretely in the tube, which helps to decrease fluid resistance by reducing surface area for disturbing flow.

Guo et al. [19] afresh surveyed the physical mechanism of convective heat transfer, and proposed field synergy principle of heat transfer enhancement on the basis of energy equation. They pointed out that convective heat transfer was affected by the synergy of velocity vector and temperature gradient in the flow field. The better the synergetic degree between velocity vector and temperature gradient is, the higher the heat transfer rate is. Liu et al. [20] extended Guo's theory, and proposed a principle of physical quantity synergy in the laminar flow field for convective heat transfer. Through improving synergetic regulation among physical quantities in the core flow, heat transfer unit with better performance can be designed.

\section{PHYSICAL AND MATHEMATICAL MODEL}

Based on the principle of heat transfer enhancement in the core tube flow, we proposed a porous medium model for making fluid temperature uniform in the laminar flow and a swirling flow model for disturbing fluid in the turbulent flow. Theoretical analysis and numerical simulation are conducted. When constructing physical models of heat transfer enhancement in the core flow, we should particularly follow the principle of reducing surface area of heat transfer component to decrease frictional resistance.

\subsection{Porous Medium Model}

Making fluid temperature uniform in the core flow region of a tube is an important way of enhancing heat transfer. To do so, porous medium with high thermal conductivity and high porosity is selected as the tube insert which does not contact the tube wall. The theoretical model of flow and heat transfer in the saturated porous media is well established in the recent literatures [21-24], which is used in our numerical calculation.

\subsubsection{Porous Model in the Fully Developed Laminar Flow}

As shown in Fig. (1), metal porous medium is filled in part of a tube without contacting the tube wall, and the laminar flow is fully developed in the tube. The tube diameter and the porous body diameter are $D$ and $d$ respectively. The effective thermal conductivity increases significantly, as the thermal conductivity of the porous medium is much higher than that of the fluid. Therefore, the temperature profile in the core flow region becomes uniform. Consequently, a relatively greater temperature gradient can be achieved near the wall, which will intensify convective heat transfer between the fluid and the tube wall.

\subsubsection{Porous Model in the Entrance Region}

When developing techniques of heat transfer enhancement, it should be noticed that there usually exists an entrance region in each tube of a heat exchanger. As shown in Fig. (2), the fluid flows through a circular tube filled with inconsecutive segments of porous medium, and each of the segments has a length $l$ without contacting the tube wall. The distance between two sections is $S$. The temperature uniformity in the core region increases, because the porous matrix has a larger thermal conductivity, and as a result, heat transfer can be enhanced between the fluid and the wall surface. The heat transfer coefficient between the fluid and the

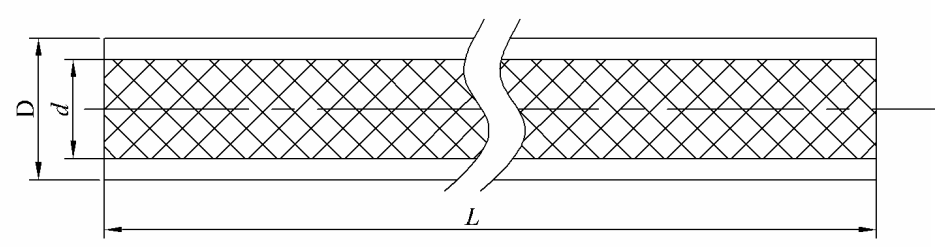

Fig. (1). Porous medium model in the fully developed laminar flow. 


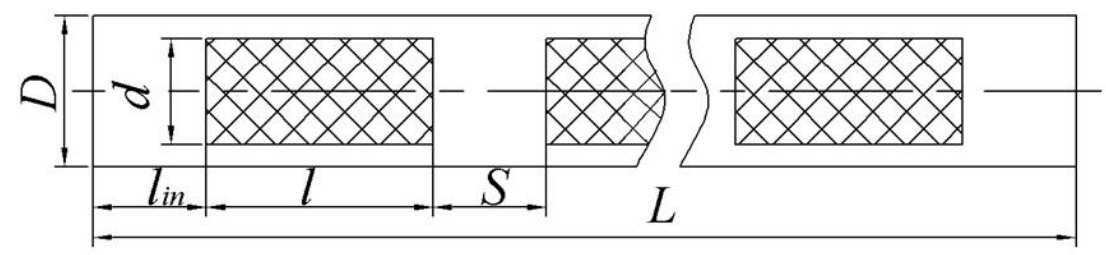

Fig. (2). Segments of porous medium in the entrance region.

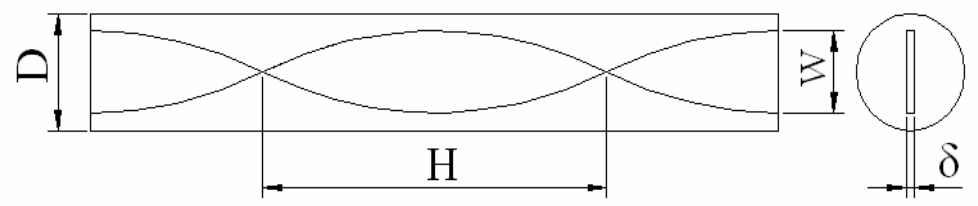

Fig. (3). Narrow twist tape model.

wall surface in the entrance section is larger than that in the fully developed section due to the effect of the thermal boundary layer. Therefore, it is benefit to reduce flow resistance and enhance heat transfer through filling the tube with segments of porous media to improve the overall performance.

\subsection{Swirling Flow Model}

The primary motive of selecting the swirling flow model is to produce swirling flow and secondary flow in the flow region which will remarkably strengthen convective heat transfer between the fluid and the tube wall. However, in order to reduce the flow resistance, it is also necessary to have a certain space between the heat transfer components and the tube wall. Concerning about this, new types of heat transfer enhanced tubes with narrow twist tapes and swirling vanes are proposed in this paper.

\subsubsection{Narrow Twist Tape Model}

As we know that there exists very large flow resistance in the turbulent flow for the heat transfer enhanced tube inserted with traditional twist tape. Consequently, the overall performances of this tube decrease greatly in the case of turbulent flow, which narrows its application. Based on the principle of heat transfer enhancement in the core flow, a model of heat transfer enhanced tube with narrow twist tape is proposed in this paper. By reducing the width of the twist tape $W$, shown in Fig. (3), the flow resistance will decrease.

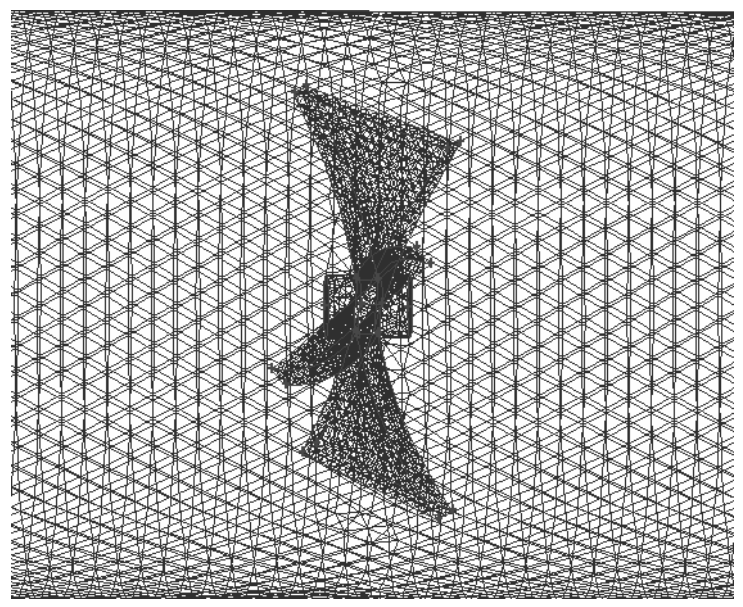

Fig. (4). Swirling vane in a tube.
For the traditional twist tape, the width is nearly equal to the tube diameter. For the narrow twist tape, however, the width is much smaller than the tube diameter.

\subsubsection{Swirling Vane Model}

An axial swirling vane component is shown in Fig. (4), which is uniformly arranged inside the core flow region of a tube with diameter $20 \mathrm{~mm}$ and length $960 \mathrm{~mm}$, and each group is connected with a slender rod. The swirling vanes will not contact with the tube wall. By carefully arranging the number of swirling vane units inside the tube, we can get a relatively higher overall performance of heat transfer enhanced tube, as in this case, convective heat transfer coefficient is large and swirling vane units do not lead to excessive flow resistance along the tube.

\section{MATHEMATICAL MODEL AND NUMERICAL CALCULATION}

\subsection{Mathematical Model}

The general form of governing equation for continuity, momentum and energy can be expressed as

$$
\begin{aligned}
& \frac{\partial(\rho u \Phi)}{\partial x}+\frac{\partial(\rho \nu \Phi)}{\partial y}+\frac{\partial(\rho w \Phi)}{\partial z}=\frac{\partial}{\partial x}\left(\Gamma \frac{\partial \Phi}{\partial x}\right)+ \\
& \frac{\partial}{\partial y}\left(\Gamma \frac{\partial \Phi}{\partial y}\right)+\frac{\partial}{\partial z}\left(\Gamma \frac{\partial \Phi}{\partial z}\right)+S
\end{aligned}
$$

where $\rho$ is fluid density; $u, v, w$ are the velocity components of fluid in $x, y$ and $z$ direction, respectively; $\Gamma$ is general diffusion coefficient; $S$ is the source item which varies in different equations and includes Darcy item and Forchheimer item in the porous medium model; $\Phi$ is a general variable: in continuity equation, $\Phi=1$; in momentum equations, $\Phi=u, v, w$, respectively; in energy equation, $\Phi=T$; for equations to describe turbulent characteristics, $\Phi=k, \mathcal{E}$, respectively. The definition of coefficients and the selection for parameters in these equations can be found in literature [25].

\subsection{Results and Analysis}

For the above mathematical model, the finite difference method is used to carry out the numerical simulation for velocity and temperature distributions. The second order up- 


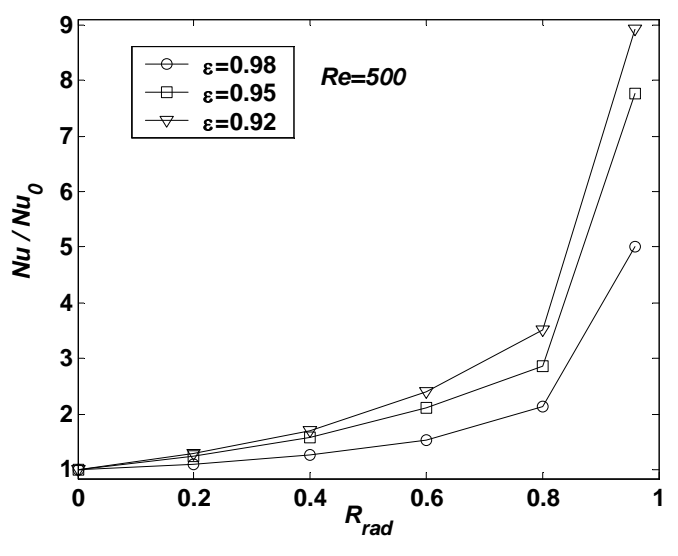

(a) Comparison of $\mathrm{Nu}$

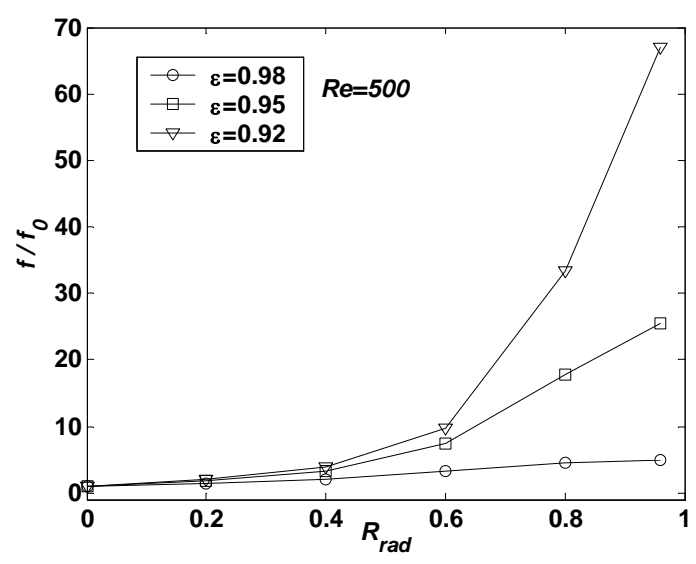

(b) Comparison of $f$

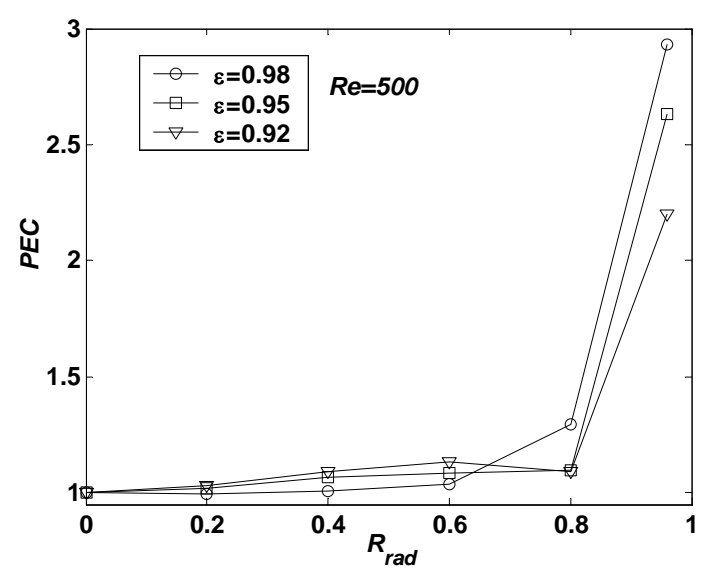

(c) Comparison of PEC

Fig. (5). Parameters of heat transfer enhanced tube filled with porous medium in the fully developed region.

wind difference is used for difference format; the QUICK method is used to solve convection item; the SIMPLE algorithm is used to couple pressure and velocity; Near-wall function method is used to describe flow and heat transfer characteristics. The convergence criterion for velocity and temperature is $10^{-8}$. For different models, the computational grid number varies. The grid number selected should meet the grid independent demand. In the simulations, water is used as working fluid.

\subsubsection{Porous Model in the Fully Developed Region}

In this porous model, the Re number is chosen as 500, the tube diameter $D$ is $20 \mathrm{~mm}$, the inlet velocity related to the $R e$ number is uniform, and the inlet temperature is $80^{\circ} \mathrm{C}$. Several assumptions are made as that the laminar flow is fully developed in the tube, the porous medium filled in the tube is a circled metal wire matrix with its thermal conductivity of $200 \mathrm{~W} /\left(\mathrm{m} \cdot{ }^{\circ} \mathrm{C}\right)$, and a constant heat flux of $310 \mathrm{~W} / \mathrm{m}^{2}$ is imposed on the tube wall.

Numerical analysis is conducted on the physical model shown in Fig. (1) and the result is shown in Fig. (5). The performance evaluation criteria is employed as

$$
P E C=\frac{N u / N u_{0}}{\left(f / f_{0}\right)^{1 / 3}}
$$

where $N u_{0}$ and $f_{0}$ are Nusselt number and friction coefficient for bare tube, respectively.

It can be seen from Fig. (5) that for porous medium with $\varepsilon=0.92$ and porous filling ratio $R_{\text {rad }}=d / D=0.95$, the $P E C$ value of heat transfer enhanced tube in the fully developed region surpasses 2 . If $\varepsilon=0.95$, the $P E C$ value can surpass 2.5 when other parameters keep unchanged.

\subsubsection{Porous Model in the Entrance Region}

In this porous model, the Re number is chosen from 200 to 2000 , the tube length $L$ is $960 \mathrm{~mm}$, the tube diameter $D$ is $20 \mathrm{~mm}$, the inlet velocity related to the $R e$ number is uniform, the inlet temperature is $20{ }^{\circ} \mathrm{C}$, and a constant heat flow of $4000 \mathrm{~W} / \mathrm{m}^{2}$ is imposed upon the tube wall. Several sections of porous media, with length $l=30 \mathrm{~mm}$ and segment spacing $S=15 \mathrm{~mm}$, have been arranged along the tube. There is an annular spacing between the porous matrix and the tube wall.

As shown in Fig. (6), there is a positive effect on heat transfer enhancement when filling porous medium in the tube discretely. Because heat transfer coefficient in the entrance region of a tube is higher than that in the fully developed region, the $P E C$ value shown in Fig. (6) is not as high as that shown in Fig. (5). When porous filling ratio is 0.95 , the $P E C$ value may surpass 1.1 in laminar flow and its highest value may be over 1.6. As the specific surface of porous medium is greatly reduced when using discrete method, flow resistance decreases, and as a result, the overall performance of heat transfer enhanced tube is improved. From Fig. (6), we can see that the $P E C$ value of tube II with porous filling ratio $98 \%$ is higher than that of tube I with porous filling ratio $95 \%$. However, the filling ratio $98 \%$ is very difficult to reach in the application. Moreover, when it reaches or surpasses this ratio, the scale effect of very small annular space between the porous matrix and the tube wall should be considered.

\subsubsection{Narrow Twist Tape Model}

In the calculation, the temperature of the tube wall is set to be constant. The torsion coefficient of the twist tape is 2.25 , the tube diameter $D$ is $20 \mathrm{~mm}$, and the filling ratio of the twist tape $W / D$ is $0.5,0.7$ and 0.9 respectively. Periodic boundary condition is imposed on the inlet and outlet of the 


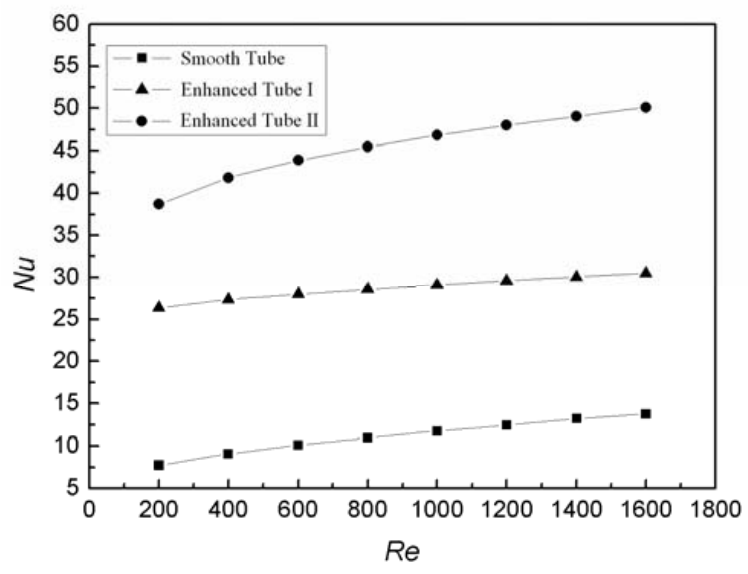

(a) Comparison of $\mathrm{Nu}$ number

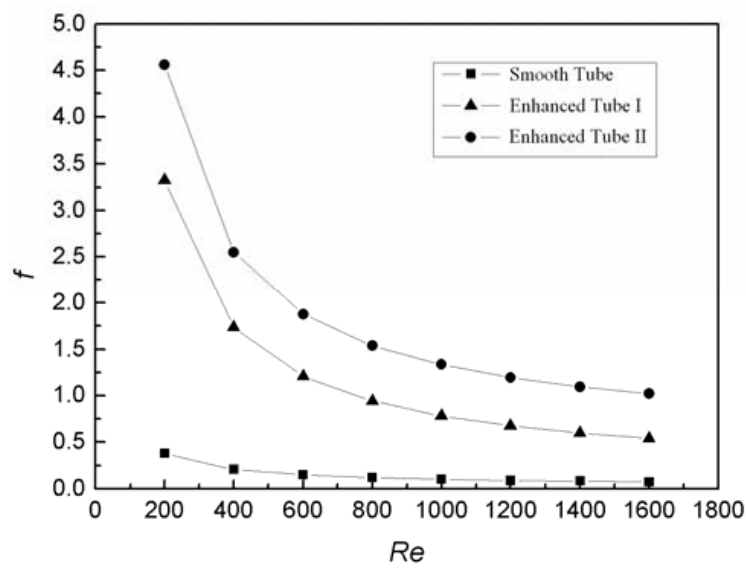

(b) Comparison of $f$

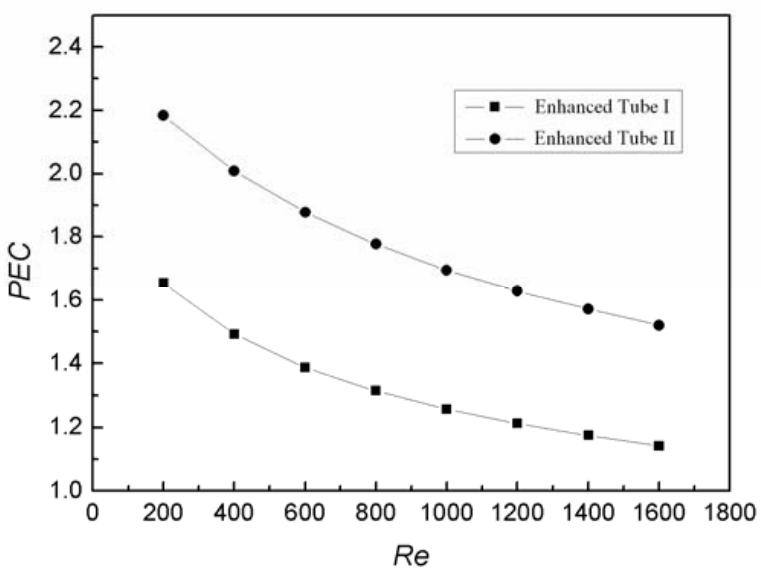

(c) Comparison of PEC

Fig. (6). Parameters of heat transfer enhanced tube filled with porous medium in the entrance region (enhanced tube I: porous filling ratio 95\%; enhanced tube II: porous filling ratio 98\%).

physical model. The total calculation grids are approximately 500000 700000.

Fig. (7) demonstrates that the performance of the tradition twist tape surpasses that of the narrow one in the laminar flow, but the traditional twist is not suitable for the turbu-

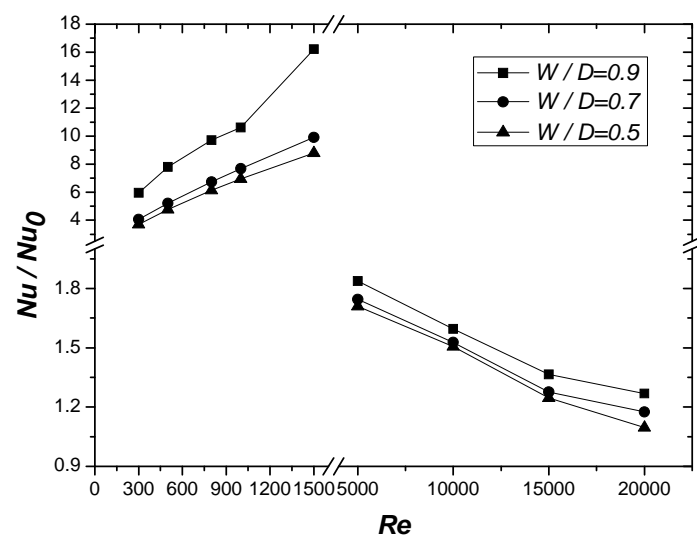

(a) Comparison of $\mathrm{Nu}$

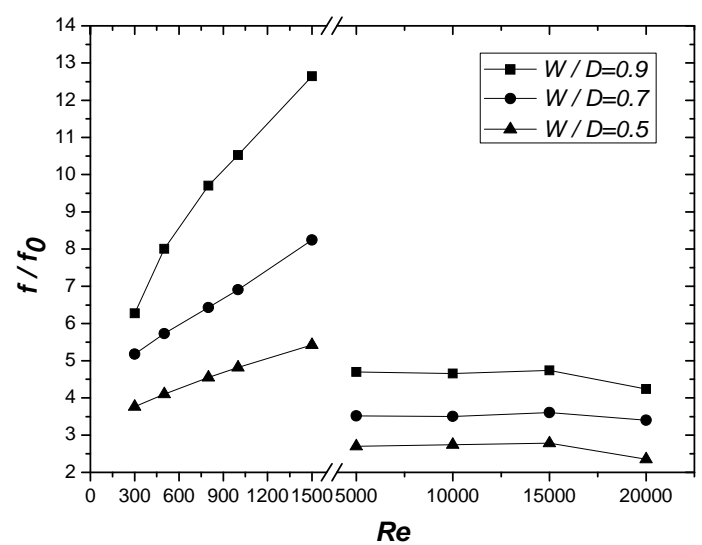

(b) Comparison of $f$

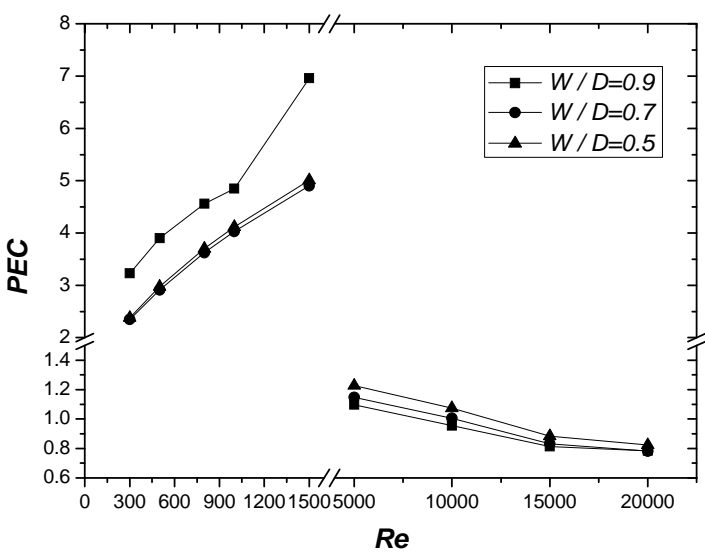

(c) Comparison of PEC

Fig. (7). Parameters of heat transfer enhanced tube filled with narrow twist tape.

lent flow. As we can see in Fig. (7), the $P E C$ value may reach 1.1 1.3 for narrow twist tape as the $R e$ number ranges in 5000 10000, but the $P E C$ value of the traditional one is only approximately $0.8 \sim 1.0$. In other words, after changing the traditional twist tape to the narrow one, the $P E C$ value 


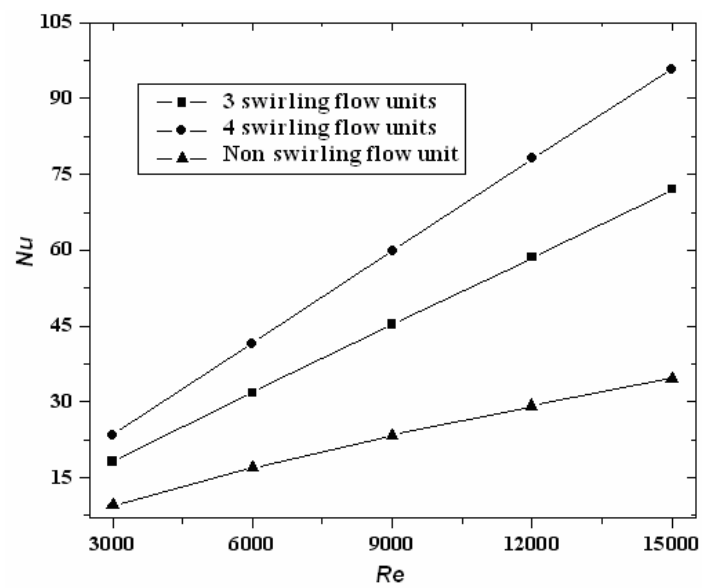

(a) Comparison of $\mathrm{Nu}$

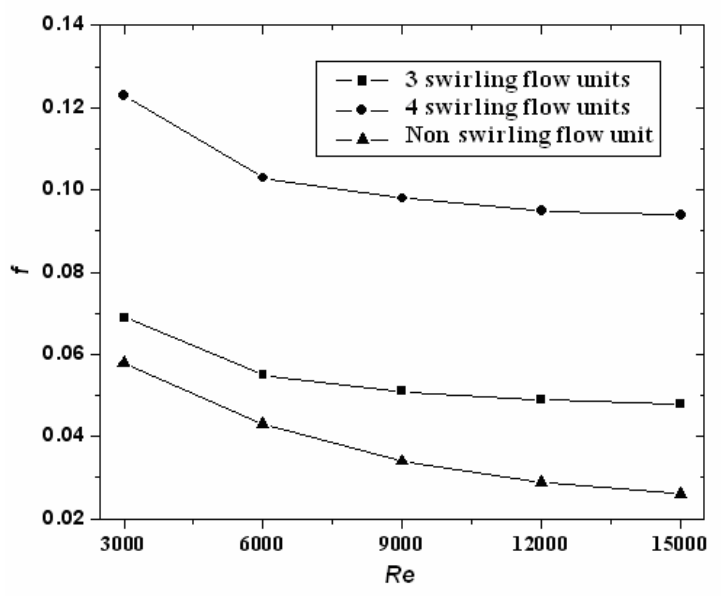

(b) Comparison of $f$

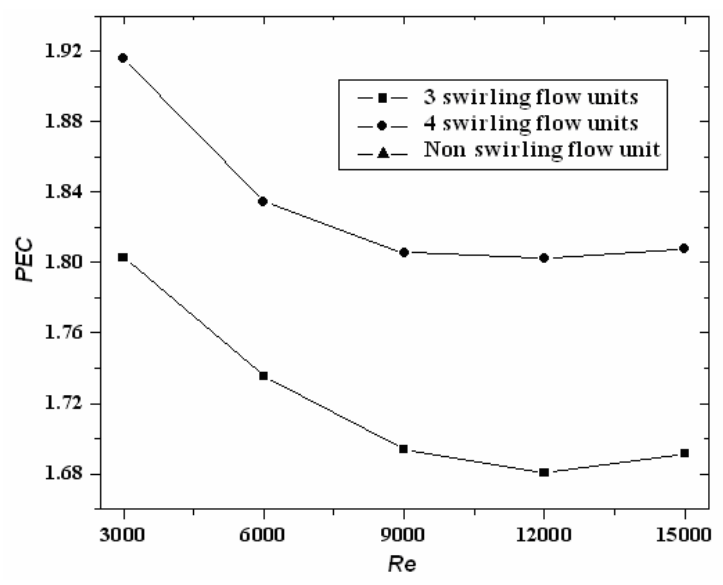

(c) Comparison of PEC

Fig. (8). Parameters of heat transfer enhanced tube filled with swirling vane.

increases more than $30 \%$. This would widen the applications by using narrow twist tape because of the decrease in flow resistance.

\subsubsection{Swirling Vane Model}

In the calculation, the Re number ranges from 3000 to 15000. The tube length is $960 \mathrm{~mm}$, the tube diameter is $20 \mathrm{~mm}$, vane thickness is $0.5 \mathrm{~mm}$, vane end width is $5 \mathrm{~mm}$, and vane height is $8 \mathrm{~mm}$. The inlet conditions are given as uniform velocity and temperature. In addition, the tube wall has a constant heat flux of $15000 \mathrm{~W} / \mathrm{m}^{2}$. The standard $k-\varepsilon$ model is used to simulate the turbulent characteristic of the tube flow.

Fig. (8) shows the simulation results for tube with 4 groups of swirling vane units with blade number 3 or 4 . It can be seen from this figure that $\mathrm{Nu}$ number and friction coefficient for the tube with 4-blade swirling vane unit are higher than those of 3-blade one. Additionally, the PEC value of the former is higher than that of the latter.

Therefore, the number of swirling vane units in the tube, as well as the amount of the blade of each unit, has great influence on the performance of heat transfer and flow resistance. In the simulated Re number range, all of the PEC values surpass 1.5 and its peak value reaches 1.9 in low $R e$ number. So, the swirling vane which effectively organizes the flow in the tube can enhance convective heat transfer and reduce flow resistance.

\section{CONCLUSIONS}

Two types of heat transfer enhancement models in the tube are proposed in this paper, and mechanisms of heat transfer enhancement in the core flow of the tube have been revealed. Some conclusions are summarized as follows.

(1) Heat transfer enhancement in the core flow of a tube is different from that in the boundary flow. Heat transfer enhancement can be achieved by making fluid temperature uniform in the core flow of a tube and reducing flow resistance.

(2) Methods of heat transfer enhancement in the core flow of a tube can be divided into two types, uniform temperature model and disturbing flow model, which are verified to be effective by the numerical simulation in this paper.

(3) According to the principle of heat transfer enhancement in the core flow of a tube, various heat transfer enhancement components can be designed. Therefore, it has a positive influence on developing heat transfer enhanced tube with better overall performance and heat exchanger with high heat transfer coefficient as well as low flow resistance.

\section{ACKNOWLEDGMENTS}

This work was supported by the National Key Basic Research Development Program of China (2007CB206903) and the Natural Science Foundation of China (50721005).

\section{REFERENCES}

[1] A. E. Bergles, "ExHFT for fourth generation heat transfer technology", Exper. Thermal Fluid Sci. vol. 26, pp. 335-344, 2002.

[2] R. L. Webb, Principles of enhanced heat transfer. Wiley: New York, 1994.

[3] F. Giampietro, "Heat transfer optimization in internally finned tubes under laminar flow conditions", Int. J. Heat Mass Transfer, vol. 41, pp. 1243-1253, 1998.

[4] P.G. Vicente, A. Garcia and A. Viedma. "Experimental investigation on heat transfer and frictional characteristics of spirally corru- 
gated tubes in turbulent flow at different Prandtl numbers [J]", Int. J. Heat Mass Transfer, vol. 47 (4) pp. 671-681, 2004.

[5] P. Naphon, M. Nuchjapo, and J. Kurujareon, "Tube side heat transfer coefficient and friction factor characteristics of horizontal tubes with helical rib", Energy Conversion Manage. vol. 47, pp. 30313044, 2006.

[6] M. Siddique and M. Alhazmy, "Experimental study of turbulent single-phase flow and heat transfer inside a micro-finned tube", Int. J. Refrigeration, vol. 31, pp. 234-241, 2008.

[7] W. Liu, K. Yang, and A. Nakayama, "Enhancing heat transfer in the core flow by forming an equivalent thermal boundary layer in the fully developed tube flow", in Sixth International Conference on Enhanced, Compact and Ultra-Compact Heat Exchangers: Science, Eng. Technol. pp. 245-250, 2007.

[8] W. Liu and K. Yang, "Mechanism and numerical analysis of heat transfer enhancement in the core flow along a tube", Sci. China, Series E, vol. 51, pp. 1195-1202, 2008.

[9] R. M. Manglik and A. E. Bergles, "Heat transfer and pressure drop correlations for twisted tape inserts in isothermal tubes: part Ilaminar flows", ASME J. Heat Transfer, vol. 115, pp. 881-889, 1993.

[10] R. M. Manglik and A. E. Bergles, "Heat transfer and pressure drop correlations for twisted tape inserts in isothermal tubes: part II transition and turbulent flows", ASME J. Heat Transfer, vol. 115, pp. 890-896, 1993.

[11] S. Ray and A. W. Date, "Laminar flow and heat transfer through square duct with twisted tape insert", Int. J. Heat Fluid Flow, vol. 22, pp. 460-472, 2001.

[12] S. W. Chang, Y. J. Jan, and J. S. Liou, "Turbulent heat transfer and pressure drop in tube fitted with serrated twisted tape", Int. J. Therm. Sci. vol. 46, pp. 506-518, 2007.

[13] S. W. Chang, T. L. Yang, and J. S. Liou, "Heat transfer and pressure drop in tube with broken twisted tape insert", Exper. Thermal Fluid Sci. vol. 32, pp. 489-501, 2007.
[14] M. Yilmaza, O. Comaklia, and S. Yapici, "Enhancement of heat transfer by turbulent decaying swirl flow", Energy Conversion Manage. vol. 40, pp. 1365-1376, 1999.

[15] L. K. Wang and B. Sunden, "Performance comparison of some tube inserts", Int. Comm. Heat Mass Transfer, vol. 29, pp. 45-56, 2002.

[16] P. Promvonge and A. S. Eiamsa, "Heat transfer enhancement in a tube with combined conical-nozzle inserts and swirl generator", Energy Conversion Manage. vol. 47, pp. 2867-2882, 2006.

[17] B. I. Pavel and A. A. Mohamad, "An experimental and numerical study on heat transfer enhancement for gas heat exchangers fitted with porous media", Int. J. Heat Mass Transfer, vol. 47, pp. 49394952, 2004.

[18] R. M. Manglik, Heat Transfer Enhancement, in Heat Transfer Handbook, A. Bejan, A. D. Kraus, Eds. New Jersey: John Wiley \& Sons, 2003.

[19] Z. Y. Guo, D. Y. Li, and B. X. Wang, "A novel concept for convective heat transfer enhancement", Int. J. Heat Mass Transfer, vol. 41, pp. 2221-2225, 1998.

[20] W. Liu, Z.C. Liu, T.Z. Ming and Z.Y. Guo. "Physical quantity synergy in laminar flow field and its application in heat transfer enhancement [J]", Int. J. Heat Mass Transfer, vol. 52 (19-20), pp. 4669-4672, 2009.

[21] D. A. Nield and A. Bejan, Convection in Porous Media. Springer: New York 2006

[22] D. B. Ingham and I. Pop, Transport Phenomena in Porous Media. Oxford: Elsevier, 2005.

[23] K. Vafai, Hanbook of Porous Media. Boca Raton: Taylor \& Francis, 2005.

[24] P. Vadasz, Emerging Topics in Heat and Mass Transfer in Porous Media. Springer: New York 2008.

[25] S. V. Patankar, Numerical Heat Transfer and Fluid Flow. McGraw-Hill: New York 1980.

(C) Liu et al.; Licensee Bentham Open.

This is an open access article licensed under the terms of the Creative Commons Attribution Non-Commercial License (http://creativecommons.org/licenses/by-nc/3.0/) which permits unrestricted, non-commercial use, distribution and reproduction in any medium, provided the work is properly cited. 